\title{
Technical Notes on GAST Final Days
}

\author{
by Jana Kadlecová - Faculty of Transportation Sciences, Czech Technical University \\ in Prague, kadlecova@lss.fd.cvut.cz
}

November 30th was the final day of the GAST project's life. The project, whose full title is Green And Safe Transportation, was financed through the EU - EC, Directorate for Education and Culture. This activity involved 18 highly professional academic and industrial partners from 7 countries and closely cooperated with three other pilot projects aimed at the innovation and improvement of the exchange of knowledge between academic and industrial areas. The importance of this quartet was primarily based on new scenarios that should show possible ways in which to accelerate the advancement of technologies accessible for all subjects needed; not necessarily to create them, but to rather find some working scheme that would enable access to information and improve its usability.

These four pilot projects, comprising of SUCCESS, BRIDGE, EIT, and, of course, GAST cooperated well and aimed at better knowledge management and the support of a "European" knowledge network.

The first task to solve was the choice of the most efficient tools to support the technology and knowledge transfer. Professionals involved in expert teams have come to the consensus to choose four test cases to prove new collaboration models, sustainable for the long-term. These test cases were developed and validated as applicable for real use. Based on the validation, several recommendations were formulated and will be presented to the European Commission as a lead for future regulation proposals.

The test case Project House dealt with the idea of a shared space where universities, industries and public bodies can meet to solve tasks that are a matter of mutual interest. Project House consists of several components that support the applied-oriented scientific activities performed. Project House has to foster innovation and technology transfer, and education and knowledge transfer by different means. The practical application will be launched in Germany and its main aim is to join the forces of universities and industries to develop or improve principles leading to lower/none carbon dioxide emissions.

Other activities that were performed were connected to the area of education, as professionals will be prepared for brand new internationally linked working principles that are a necessary prerequisite for successful future cooperation.

One of these was the Project Workshop, an international workshop of students of different educational origin who gather together in order to solve some specific task. This activity can teach its members how to cooperate using communication tools and to be responsible for teamwork's result.

The European Automotive Master study programme is a slightly moderated multiple degree study programme that offers the possibility to learn with excellence. Each member university offers the subject matter they are leaders in and there is a board to evaluate the validation of each of the members.

The last case study was aimed at the networking of currently existing separated clusters. This activity is titled Cluster of Clusters and should assure the sustainable cooperation of clusters of similar interest. As an example, the working team states EASN as a network joining clusters and facilitating the flow of knowledge between different European hubs.

As a result, there were some recommendations given for the legislative bodies and probably even for universities that are responsible for the creation and management of their study 
programmes, as these need to give the students elementary preparation in order to be able to take part in such activities.

All of the test cases performed showed the need of partial physical co-location and interaction for their long-term workability. This means that for any of the collaborative activities the "meeting" of participants is a key factor for success. Another important enhancement is provided by the good balance of private and public funding, and governance. Governance should mirror the bodies and their interests involved in projects in order for balance to be kept for all members; GAST has applied a four-layer approach.

One of the applications which are already known and that came into existence recently are KICs, which have not completely started operation, but are being prepared for launch at the moment.

What seems to be crucial in any academic-industry co-operation is the person acting as the interface; this person raises the benefits of all involved. On the other hand, the commitment of members in supporting the mutual interest is another key part.

Altogether, along with partner projects, GAST has shown that international cooperation is an inevitable part of future professional work in the automotive industry, either in development or in mass production.

Currently, the EC is taking action leading towards the introduction of new schemes of cooperation within Europe, and hopefully will respect the recommendations resulting from these four activities. 